Rev Biomed 2001; 12:27-34.

\title{
Revisión de una enfermedad poco conocida: la ciguatera.
}

Revisión

Heriberto Arcila-Herrera ${ }^{1}$, Julio Mendoza-Ayora ${ }^{2}$, María F. González-Franco ${ }^{1}$, Landy MonteroCervantes $^{1}$, Angel Castelo-Navarrete ${ }^{3}$.

${ }^{1}$ Centro de Investigaciones Regionales “Dr. Hideyo Noguchi”. Universidad Autónoma de Yucatán. ${ }^{2}$ UMF No. 59 Instituto Mexicano del Seguro Social, Mérida, Yucatán, México. ${ }^{3}$ Clínica Bonampak, Cancún, Q. Roo., México.

\section{RESUMEN.}

La ciguatera es uno de los padecimientos relacionados con la ingestión de toxinas marinas. Las toxinas son producidas por el dinoflagelo Gambierdiscus toxicus, que al ser consumido por pequeños peces herbívoros, viaja a través de la cadena alimentaria hasta los grandes peces depredadores. De las varias toxinas presentes, la mejor conocida es la ciguatoxina que es estable al calor y a la congelación. La ciguatoxina es un poliéter, agonista de los canales de sodio. En el último decenio se han consignado cientos de casos de ciguatera. Nosotros creemos que la incidencia de esta entidad está en aumento en la península de Yucatán, México. El cuadro clínico se caracteriza por manifestaciones gastrointestinales, neurológicas y cardiovasculares y en este orden, tanto desde el punto de vista de su aparición, en el tiempo, como por su severidad. Al inicio, uno de los principales problemas es la deshidratación y choque producidos por la severa diarrea y los vómitos, por lo que la restitución del equilibrio hidroelectrolítico es imperativa. La administración de soluciones de manitol, por vía intravenosa, constituye la principal indicación dentro de las primeras 24 horas. Las manifestaciones neurológicas están principalmente relacionadas con parestesias. La amitriptilina y la tocainida son efectivos en el tratamiento de las parestesias. La ciproheptadina es el tratamiento recomendado para el prurito y la fluoxetina se ha usado con éxito como tratamiento de la fatiga crónica.

(Rev Biomed 2001; 12:27-34)

Palabras clave: Ciguatera, toxinas marinas, toxicología, Gambierdiscus toxicus.

Solicitud de sobretiros: Dr. Heriberto Arcila-Herrera, Calle 59-A No. 552 (82 x 84), C.P. 97000, Mérida, Yucatán, México. Recibido el 10/Abril/2000. Aceptado para publicación el 7/Julio/2000. 


\section{H Arcila-Herrera, J Mendoza-Ayora, MF González-Franco y col.}

\section{SUMMARY.}

Review of a litle-known disease: the ciguatera.

Ciguatera is a toxin-related disease caused by the ingestion of a variety of toxic fish living in tropical and subtropical areas. Humans are poisoned by eating fish intoxicated with toxins generated by the dinoflagellate Gambierdiscus toxicus associated with coral reefs. The first fish to show toxicity are the herbivorous forms and ciguatoxin becomes concentrated in large carnivores by way of the marine food web. Ciguatoxin, the major active principle in ciguatera, is believed to be a lipid soluble polyether compound, that increases intracelullar transportation of sodium ions. Several thousand cases have been reported over a 10-year period. Cases are increasing in number in the yucatecan peninsula of México. Ciguatera produces gastrointestinal characteristics, neurological and cardiovascular symptoms in this order of severity and time-related presentation in the patients. For most of the patients the earliest manifestations are diarrhea and vomiting. Fluid and electrolyte replacement is necesary to treat hypotension caused by prolonged vomiting and diarrhea. Intravenous mannitol has been reported as a successful treatment for patients within 24 hours of the onset the clinical manifestations. Neurological symptoms involve mainly paresthesias. Amitriptyline has proven effective for paresthesias as has tocainida and cyrproheptadine for pruritus. The use of fluoxetine is successful in the treatment of chronic fatigue.

(Rev Biomed 2001; 12:27-34)

Key words: Ciguatera marine toxins, toxicology, Gambierdiscus toxicus.

\section{INTRODUCCIÓN.}

La relación del hombre con el mar es importante para la sobrevivencia del primero y la conservación del segundo. Cada día dependemos más de las fuentes de alimentación de origen marino, por lo que el conocimiento de esta relación es necesario, especialmente en cuanto se refiere a sus potenciales riesgos.

Los padecimientos relacionados con la ingestión de toxinas marinas se han agrupado de la siguiente manera $(1,2)$ :

1.- La intoxicación paralizante producida por la ingestión de mariscos bivalvos, animales filtradores que se alimentan del fitoplancton suspendido en el agua.

2.- La intoxicación producida por la tetrodotoxina del pez globo (puffer fish, fugo de los japoneses).

3.- La enfermedad neurotóxica producida por el consumo de mariscos, que es debida al dinoflagelo Tymnodinium breve, que con frecuencia es responsable de la "marea roja".

4.- La intoxicación diarreica producida por mariscos, cuyos síntomas se confunden fácilmente con la gastroenteritis infecciosa.

5.- La ciguatera.

La ciguatera es el tipo más frecuente de intoxicación debida al consumo de carne de pescado, de escama y aleta, con toxinas. Resulta de ingerir ciertos pescados que contienen toxinas que viajan a través de la cadena alimentaria. Se caracteriza por manifestación gastrointestinales, neurológicas y cardiovasculares y en este orden, tanto desde el punto de vista de su aparición en el tiempo, como por su severidad (3-5).

Fue descrita en 1606 en el Pacífico Sur. El cuadro clínico se consignó en 1774 en Nueva Caledonia por el navegante inglés, el capitán James Cook, quien describió los síntomas clínicos observados en su tripulación afectada, que coinciden con los hoy conocidos. Además, las vísceras de los pescados ingeridos por la tripulación fueron dadas a los cerdos, a los que causó la muerte (6). El término ciguatera se originó de una voz caribe usada al describir la intoxicación producida por la ingesta del caracol marino turba pica (llamada cigua). Esta descripción la hizo en la Habana el biólogo portugués, Don Antonio Parra en 1787 (7). De esta manera, ciguatera y

\section{Revista Biomédica}




\section{La ciguatera.}

ciguatoxina son, en la actualidad, dos palabras de origen caribe que pertenecen al lenguaje científico internacional.

El calor y la congelación no afectan a las toxinas y el cuadro clínico puede hacerse presente en todo lugar y momento, aún en lugares lejanos a las costas en los que el pescado, con las toxinas, puede ser llevado para su consumo (8). De esto deriva nuestro interés: el que esta entidad sea conocida por todo el grupo médico, aunque el ejercicio profesional se realice en sitios lejanos a la costa.

\section{ETIOLOGÍA.}

Las toxinas responsables de la enfermedad son producidas por el dinoflagelo que se adhiere a las superficies del coral muerto y a las algas del fondo marino. Junto con la hierba marina, son consumidos por pequeños peces herbívoros y éstos, a su vez, sirven de alimento a peces carnívoros de mayor tamaño, lo que condiciona que las toxinas del dinoflagelo viajen por la cadena alimentaria hasta llegar a los grandes peces depredadores como lo es la barracuda. De los cientos de variedades del fitoplancton, que es la base de la cadena alimentaria, aproximadamente una docena de ellos son tóxicos entre los que se cuentan los dinoflagelos, los primesiofitos y los cloromonados $(8,9)$. Entre los dinoflagelos, principalmente el Gambierdiscus_toxicus es el primeramente relacionado con la ciguatera (10). Eventualmente estas algas pueden florecer en gran abundancia y se convierten, con la luz solar, en biomasa. Un mililitro de agua de mar puede contener miles de algas tóxicas que se expanden a amplias zonas del mar dándole el color rojo a café, de donde deriva el nombre de "marea roja" (11). En condiciones ambientales determinadas, las algas pueden tomar la forma enquistada y así vivir durante años. Condiciones naturales como tormentas y huracanes modifican la ecología y es cuando estas algas tóxicas florecen. Estos cambios también pueden ser inducidos por el hombre como sucede en los naufragios, establecimiento de fondeadores, puertos de abrigo, prácticas de bombardeo, operaciones de minería y dragado, construcciones y blancos militares. Estas son algunas de las actividades llevadas por el hombre que pueden alterar importantemente la ecología (12).

\section{TOXICOLOGÍA.}

Son varias las toxinas encontradas en una amplia variedad de pescados (13). Aparte de la ciguatoxina, se han identificado a la escaritoxina, la maitotoxina, la politoxina y el ácido okadaico (14-16). Sólo la ciguatoxina y la maitotoxina han sido aisladas del G. toxicus. Existe la posibilidad que el dinoflagelo contenga precursores menos polares de la toxina y que ya en el pez, las toxinas sean oxidadas a formas más polares y por ende, más tóxicas (17).

La ciguatoxina se ha identificado como un poliéter, con un nitrógeno cuaternario, estable en el jugo gástrico y resistente al calor y a la congelación $(8,18)$. Su molécula se fija a la bicapa lipídica de la membrana celular (19). Se une y abre los canales de sodio, permitiendo la entrada de este ión a la célula (20). La presencia de mayor sodio intracelular, ejerce fuerza osmótica la que produce mayor ingreso de agua intracelular e hinchazón de las células de Schwan y de los axones (21). En estas condiciones, cuando llegan nuevos estímulos existe salida del sodio intracelular en exceso, en intercambio con calcio extracelular (22). Finalmente, las células tienen mayor concentración de calcio, el que actuando como segundo mensajero condiciona anormalidades en las células del epitelio intestinal, terminando en diarrea (23). La ciguatoxina también evoca potenciales de acción espontáneos en el músculo estriado y en el miocardio (24).

La maitotoxina es también un poliéter soluble en agua (17). Experimentalmente se ha demostrado que es más tóxica que la ciguatoxina. La maitotoxina actúa en presencia de la tetrodotoxina (un bien conocido bloqueador de los canales del sodio), lo que hace improbable que su acción sea sobre los canales del sodio. Se ha propuesto que

Vol. 12/No. 1/Enero-Marzo, 2001 


\section{H Arcila-Herrera, J Mendoza-Ayora, MF González-Franco y col.}

es un agonista de los canales del calcio (25). Aunque la maitotoxina produce aumento del calcio intracelular, se ha demostrado que actúa aún en presencia de la nifedipina y el verapamilo (conocidos calcio-antagonistas). Por lo anterior, se piensa que la maitotoxina activa a alguna enzima membranal, la que inicia la cascada de la proteína $\mathrm{G}$, con la liberación final del calcio del retículo endoplásmico hacia el citosol (26).

\section{EPIDEMIOLOGÍA.}

Esta entidad ocurre en un cinturón que va alrededor del mundo entre las latitudes $35^{\circ}$ norte y $35^{\circ}$ sur, en el que queda incluido el caribe, las penínsulas de Yucatán y La Florida, las islas hawaianas, la Polinesia Francesa y Australia (9). El caribe y las islas Indo-Pacíficas son consideradas como áreas endémicas (10).

De 1972 a 1977 se consignaron en los Estados Unidos 55 casos de ciguatera (11). En Miami la incidencia anual estimada fue de 50 casos por diez mil habitantes (27). En Hawai fue de 8.7 casos por diez mil habitantes, de 1984 a 1989 (28). En las Islas Caimán y en la Martinica, en 1981, se consignó la incidencia de 43 y 41 casos por diez mil habitantes, respectivamente (29). En Japón se consignaron 379 casos de 1949 a 1980 (11). En Australia se identificaron 479 casos de 1978 a 1984 (30).

Esta enfermedad es conocida en todas las costas de la república mexicana (31). En la península de Yucatán siempre ha existido y la conocemos por relatos anecdóticos. Entre la gente de mar se le conoce como "ciguata" y se dice que se presenta en los meses en cuyo nombre no está la letra " $r$ "; esto es, durante el tiempo de temporales y ciclones. A partir de una experiencia de nuestro grupo con 10 casos de ciguatera (3), hemos entrevistado a médicos, pescadores y personas expertas en puertos marinos de la península y tenemos la impresión de que en el último quinquenio se incrementó el número de casos entre los meses de mayo y agosto de cada año. Lo anterior ha sido especialmente en los estados de
Yucatán y Quintana Roo; no hemos sido informados de algún caso en el estado de Campeche. Nuestra información es anecdótica y no sabemos si esto es un hecho real o se debe a que la gente está más informada y advertida.

Existe, entre los pescadores, todo un cuerpo de conocimiento empírico: se dice que la barracuda que no pelea cuando se le tiene cogida en la línea, ya está enferma. También se detecta la enfermedad porque al corte, la carne del pescado no está firme, sino "masuda" y su color es violáceo. Se puede limpiar monedas de cobre con el jugo de la carne y finalmente, las vísceras del pescado se le dan a comer a los perros y éstos amanecen muertos al día siguiente.

\section{CUADRO CLÍNICO Y TRATAMIENTO.}

Los signos y síntomas gastrointestinales son los que predominan en el cuadro clínico por su severidad y por ser los más tempranos en presentarse. Los pacientes tienen diarrea líquida abundante y vómitos, que rápidamente pueden llevarlos a la deshidratación y choque $(2,5,23$, 31). En estos casos, la reposición inmediata de agua y electrólitos es imperativa. Dos de los pacientes, por nosotros estudiados, cayeron en deshidratación y choque y su recuperación se debió a su pronto tratamiento. También pueden existir hipersalivación, náusea y dolores abdominales (3). Se ha sugerido que las toxinas estimulan directamente la secreción de fluido enteral sin existir ningún daño tisular y que este proceso de secreción está mediado por el calcio como segundo mensajero. El diagnóstico se hace clínicamente, todas las pruebas de laboratorio de rutina son normales y no existe una prueba de laboratorio o gabinete confirmatoria del diagnóstico. La hipertermia y el síndrome febril no existen, toda vez de que no se trata de una enfermedad infecciosa, como tampoco existe desviación de la cuenta leucocitaria ni de su diferencial. Esto hace el diagnóstico diferencial con otras entidades que cursan con diarrea acentuada (32).

Lo que es distintivo en esta entidad son las

\section{Revista Biomédica}




\section{La ciguatera.}

manifestaciones neurológicas, especialmente la hipersensibilidad al frío y la inversión de las sensasiones calor/frío. Pueden existir parestesias y alodinia en las extremidades y en los labios, cefalea, mareos, vértigos, ataxia, temblores, rigidez del cuello, sensación de dientes flojos, hipersensibilidad en pezones que solamente ha sido consignada en mujeres, depresión, artralgias y mialgias. Estas manifestaciones pueden ser tempranas y habitualmente siguen, en un tiempo inmediato, a las manifestaciones gastrointestinales $(5,27,33)$. Se ha consignado que la administración de gluconato de calcio revierte estos síntomas neurológicas de la ciguatera (33). Se ha descrito el caso de un paciente, que después de ingerir una sopa de pescado inició el cuadro clínico con manifestaciones gastrointestinales y neurológicas, para después progresar al estado de coma (34).

Se ha indicado ampliamente el uso del manitol, por vía intravenosa, en el curso de las primeras 24 horas de iniciado el cuadro clínico. $\mathrm{Su}$ beneficio se relaciona con su capacidad osmótica, ya que puede actuar sobre el edema axonal y encefálico. Como diurético podría acarrear toxinas por la orina. También, como la ciguatoxina es rica en grupos oxidrilo, que se fijan al manitol, entonces éste actúa como un efectivo removedor de radicales libres $(35,36)$.

Se ha demostrado que las sensaciones, descritas por los pacientes como "piquetes", "dolor profundo", "entumecimiento", "quemadura" y "toque eléctrico" son generadas por fibras nociceptivas $\mathrm{C}$-polimodales tanto de la piel como de tejidos profundos, y que la intensidad de estas sensaciones depende de la intensidad de descarga de estas mismas fibras (37-39). Cameron pone en duda la sensación paradójica de la temperatura, también conocida como inversión calor/frío. Estudia a pacientes sumergiéndoles las manos en agua que está a temperaturas entre 0 y $50^{\circ} \mathrm{C}$. Sugiere que esta sensación paradójica no es más que un resultado exagerado de sensibilidad, sin que exista confusión de las sensaciones calor/frío (40).

Se recomienda el diazepán y la carbamazepina para las convulsiones y la ansiedad (4). Indometacina para el dolor articular, tocainida para las parestesias y la ciproheptadina para el prurito (9). Igualmente se ha consignado el uso del ácido ascórbico y la vitamina $\mathrm{B}$ para la sintomatología neurológica y los opiacios para casos de dolor extremo (44). La amitriptilina, primeramente indicada para el tratamiento de la depresión, ha mostrado efectividad en las manifestaciones neurológicas de la entidad; aumenta la cantidad de norepinefrina en el sistema nervioso central y regula a los canales del sodio $(9,32,41)$. Se ha consignado, igualmente, el uso exitoso de la fluoxetina en la fatiga crónica a que lleva la enfermedad (42).

Las principales manifestaciones cardiovasculares son la bradicardia y la hipotensión arterial. En Hawai estudiaron a 15 pacientes que ingirieron pescado con toxinas y la ciguatoxina fue identificada en los sobrantes de la comida. Diez de los pacientes tuvieron bradicardia y dos de ellos llegaron a terapia intensiva. La bradicardia se asoció a la mayor edad de los pacientes y a mayor cantidad de toxina ingerida. La mayor duración del cuadro clínico se asoció a mayor edad y peso de los pacientes, pero no con la cantidad de la toxina ingerida (43). En otro estudio, se consigna la presencia de bradicardia en el $14 \%$ de los pacientes y de hipotensión arterial en el $2 \%$ de los mismos (5). Se ha observado que la ciguatoxina primero produce una fase de hipotensión y bradicardia que puede ser abolida con el uso de atropina y una segunda fase de hipertensión y taquicardia que puede ser bloqueada con reserpina y propanolol. La primera fase es sugestiva de activación directa de canales de sodio dependientes de voltaje $(44,45)$. Se ha consignado el caso de un paciente de 53 años de edad que presentó hipotensión ortostática posterior a la ingestión de barracuda con el tóxico de la ciguatera. Los autores piensan que la hipotensión fue debida a tanto insuficiencia simpática como a insuficiencia parasimpática (46).

Los pacientes también acusan debilidad, boca seca, prurito en palmas y plantas. Algunos pacientes se quejan de disuria y las molestias se 


\section{H Arcila-Herrera, J Mendoza-Ayora, MF González-Franco y col.}

acentúan en ocasión de ingestión de alcohol, de comer pescaso sin toxinas y con la actividad sexual $(4,47)$. Se ha encontrado midriasis, visión borrosa, fotofobia y ceguera transitoria (48). Los síntomas habitualmente se inician entre la tercera y la quinta hora después de la ingestión del pescado con las toxinas. En nuestro estudio el período más corto de inicio fue de 20 minutos y el más prolongado fue de 12 horas (3). El cuadro clínico dura habitualmente de dos a tres semanas y se habla de una fase aguda que puede prolongarse hasta 6 meses, y cuando dura más de este tiempo se dice que el padecimiento se ha hecho crónico (5). La edad promedio en que se presentó el síndrome en un estudio de 551 pacientes, en la Polinesia Francesa, fue de $36.6 \pm 15.6$ años. El cociente por sexo $(\mathrm{M} / \mathrm{F})$ fue de 1.6. La mayor severidad del cuadro clínico se observó cuando el pescado consumido era carnívoro y se trataba de un segundo ataque de la enfermedad (49). Los casos en que el paciente falleció estuvieron relacionados a estado comatoso. R. Bagnis (5) describe el caso de un paciente de 54 años que inició su sintomatología antes de haber terminado de comer el hígado de un pescado que había asado, falleció cinco días después en estado de coma y en la autopsia encontraron que el hígado tenía lesiones necróticas con infiltrado eosinófilo. Otro paciente de 42 años falleció al sexto día de evolución, también en estado de coma. Una niña de once años inició el cuadro 12 horas después de la ingestión del pescado, tuvo diarrea, parestesias y prurito; 99 horas después tuvo parálisis facial derecha y convulsiones de hemicuerpo derecho, tuvo rigidez e hiperreflexia; se diagnóstico decorticación cerebral y falleció después de haber estado en coma durante 20 días. E. Stommel (50) documenta dos casos, hombre y mujer, en quienes el cuadro de ciguatera fue previo al inicio de polimiositis. Esta asociación señala probable causalgia entre ambas entidades. Se sugiere que la ruptura de la fibra muscular puso al descubierto proteínas intracelulares, lo que inició reacciones de sensibilidad con posterior ataque al músculo por parte de anticuerpos y linfocitos

\section{Revista Biomédica}

sensibilizados. Las toxinas responsables del cuadro no son teratogénicas y en los casos en que ha ocurrido durante el embarazo, los productos han sido normales (51). La toxina puede ser secretada en la leche materna (52).

\section{REFERENCIAS.}

1.- Juranovic LR, Park DL. Foodborne toxins of marine origin: ciguatera. Reviews of enviromental contamination and toxicology. New York: Springer-Verlag; 1961. p. 5182.

2.- Hughes JM, Merson MH. Fish and shellfish poisoning. N Engl J Med 1976; 295:1117-20.

3.- Arcila-Herrera H, Castelo A, Mendoza J, Montero L, González F, Brito W. Diez casos de ciguatera en Yucatán. Rev Invest Clin 1998; 50:149-52.

4.- Lange WR, Snyder FR, Fudala PJ. Travel and ciguatera fish poisoning. Arch Intern Med 1992; 152:2049-52.

5.- Bagnis R, Kibenski T, Laugier S. Clinical obsrvations of 3,009 cases of ciguatera (fish poisoning) in the south pacific. Am J Trop Med Hyg 1979; 28:1067-73.

6.- Cook J, Furneayx T, Jodoes W. A voyage towards the south pole and around the world. Chap 2, $3^{\text {rd }}$. London: English Press. 1978. p. 112-13.

7.- Lange WR. Ciguatera Toxicity. Am Fam Phy 1987; 35:177-82.

8.- Beadle A. Ciguatera fish poisoning. Milit Med 1997; 162:319-22.

9.- Swift AEB, Swift TR. Ciguatera. Clin Toxicol 1993; 31:1-29.

10.- Barton E, Tanner P, Turchen SE, Tunget CL, Monoguerra A, Clark RF. Ciguatera fish poisoning. A wouther California epidemic. West J Med 1995; 163:31-5.

11.- Glaziou P, Legrand AM. The epidemiology of ciguatera fish poisoning. Toxicon 1994; 32:863-73.

12.- Ruff TA. Ciguatera in the pacific: A lind with military activities. Lancet 1989; 1:201-5.

13.- Banner AH, Ciguatera: a disease from coral reef fish. En: Jones OA, Endean R, Ed. Biology and geology of coral 


\section{La ciguatera.}

reef.. Vol. III. Nueva York: New York Acad Press; 1976. p. 177-213.

14.- Palafox NA, Jain LG, Pizano Az. Successful treatment of ciguatera fish poisoning with intravenous mannitol. JAMA 1988; 259:2740-2.

15.- Kodama AM, Hokama Y. Yasumoto T. Short communication. Clinical and laboratory findings implicating polytoxin as a cause of ciguatera poisoning due to Decapterus macrosoma (mackerel). Toxicon 1989; 27:1051-2.

16.- Moore Re, Schever PJ. Palitoxin: A nex marine toxin from a coelenterate. Science 1971; 172:495-8.

17.- Holmes MJ, Lewis RJ, Poli MA, Gillespie NC. Strains dependant production of ciguatoxin precursors (gambier toxins) by G. Toxicus (Dinophyceae) in culture. Toxicon 1991; 29:761-75.

18.- Schver PJ, Takahashi W, Tusutsmi J, Yoshida T. Ciguatoxin: isolation and chemical nature. Science 1967; 155:1267-8.

19.- Nukina M., Koyanagi LM, Schver PJ. Two interchangeable forms of ciguatoxin. Toxicon 1984; 22:16976.

20.- Molgo J., Comella JX, Legrand AM. Ciguatoxin enchances quantal transmitters release from frog nerve terminals. Br J Pharmaocol 1990; 99:695-700.

21.- Terao K, Ito E, Oarada M, Ishibashi Y. Light and electron microscopic studies of pathologic changes induced in mice by ciguatoxin poisoning. Toxicon 1991; 29:63343.

22.- Lewis RJ, Endran R. Direct and indirect effects of ciguatoxin on guinea-pig atria and papillary muscules. Arch Pharmacol 1986; 334:313-22.

23.- Fasano A, Hokama Y, Russel R, Morris JG. Diarrhea in ciguatera fish poisoning: preliminary evolution of pathophysiological mechanisms. Gastroenterology 1991; 100:471-6.

24.- Legrand AM, Galdnnier M, Bagnis R. Studies on the mode of action of ciguatera toxin. Toxicon 1982; 20:3115 .

25.- Takayashi M, Ohizumi Y, Yasumoto T, Maitotoxin a CA2+ channel activator candidate. J Biol Chen 1982;
257:7287-9.

26.- Sertino MA, Delahunty TM, Yasumoto T, Cronin MJ. Maitotoxin increases inositol phosphates in rat anterior pituitary cells. J Mol Endocrinol 1991; 6:95-9.

27.- Lawrence DN, Enriquez MB, Lumish RM. Ciguatera fish poisoning in Miami. JAMA 1980; 244:254-8.

28.- Anderson BS, Sims JK, Wiebenga NH, Sugi M. The epidemiology of ciguatera fish poisoning in Hawai 19751981. Hawai Med 1983; 42:326-34.

29.- Morris JG, Lewin P, Smith CW, Blake PA, Schneider R. Ciguatera fish poisoning. Epidemiology of the desease on St. Thomas, US, Virgen Islands. Am J Trop Hyg 1982B; 31:574-8.

30.- Guillespi NC, Lewis RJ, Pearn JH, Bourke ATC, Holmes MJ, Bourke JB, et al. Ciguatera in Australia. Occurence, clinical features, Pathophsyology and management. Med J Aust 1986; 145:584-90.

31.- Sierra-Beltran AP, Cruz A, Nuñez E, Del Villar LM, Cerecero J, Ochoa JL. An overview of the marine food poisoning in Mexico. Toxicon 1998; 36:1493-502.

32.- Lange WR. Ciguatera fish poisoning. Am J Fam Physich 1994; 50:579-84.

33.- Morris JG, Lewin P. Hargrell NT, Smith CW, Blake PA. Schnaider R. Clinical feature of ciguatera fish poisoning: A study of the desease in the U.S. Virgen Islands. Arch Intern Med 1982; 142:1090-2.

34.- De Fusc DJ, O’Dowd P, Hokama Y, Ott BR. Coma due to ciguatera poisoning in Rhode Island. Am J Med 1993; 95:240-3.

35.- Palafox NA, Jain LG, Pizano AZ, Gulick TM, Williams RK, Schatz IJ. Successful treatment of ciguatera fish poisoning with intravenous mannitol JAMA 1988; 259:2740-2.

36.- Pearn JH, Lewis RJ, Ruff T, Tait M, Quinn J, Murtha W. Ciguatera and mannitol: Experience with a new treatment regime. Med J Aust 1989; 151:77-80.

37.- Bessou P, Pearl ER. Response of cutaneous sensory units with unmyelinated fibers to noxious stimuli. J Neurophysiol 1969; 32:1025-43.

38.- Torebjork HE. Afferent $\mathrm{C}$ units responding to Vol. 12/No. 1/Enero-Marzo, 2001 


\section{H Arcila-Herrera, J Mendoza-Ayora, MF González-Franco y col.}

mechanical, thermal and chemical stimuli in human nonglabrous skin. Acta Physiol. Scand 1974; 92:374-90.

39.- Gybels J, Handwerker HO, Van Kees J. A comparison between the discharges of human nociceptive nerve fibers and the subject's rating of his sensations. J Physiol 1979; 292:193-206.

40.- Cameron J, Capra MF. The basis of the paradoxical disturbance of temperature perception in ciguatera poisoning. Clin Toxicol 1993; 31:571-9.

41.- Bowman PB. Amitriptyline and ciguatera. Med J Aust 1982; 1:57-8.

42.- Berlin RM, King SL, Blythe DG. Symptomatic improvement of chronic fatigue with fluoxetine in ciguatera fish poisoning. Med J Aust 1992; 157:2131-3.

43.- Zatz Ar, Terrell PS, Sasaki DM. Ciguatera on Kauai: investigation of factors associated with severity of illness. Am J Trop Med Hyg 1993; 49:448-54.

44.- Miyahara JT, Shibata S. Effects of ciguatera toxin on the guinea pig taenia caecum Fed Proc 1984; 35:842-4.

45.- Miyahara JT, Akau CK, Yasumoto T. Effects of ciguatoxin and maitotoxin on the isolated guinea pig atria. Res Commun Chem Pathol Pharmacol 1997; 25:177-80.

46.- Geller RJ, Benowitz NL. Orthostaatic hypotension in ciguatera fish poisoning. Arch Intern Med 1982; 152:21313.

47.- Lange WR, Lipkin KM, Yang GC. Can ciguatera be a sexually transmitted disease? J Toxicol Clin Toxicol 1989; 27:193-7.

48.- Huges JM. Marson MH. Current concepts, fish and shellfish poisoning. N Engl J Med 1976; 295:1117-20.

49.- Glaziou P, Martin PM. Study of factors that influence the clinical response to ciguatera fish poisoning. Bull Soc Pathol Exot 1992; 85:419-20.

50.- Stommel E, Parsannet J, Kenkyn L. Polymiositis after ciguatera toxin exposure. Arch Neurol 1991; 48:874-7.
51.- Senecal PE, Osterlich JD. Normal fetal outcome after maternal ciguateric toxin expose in the second trimesster. J Toxicol Clin Toxicol 1991; 29:437-78.

52.- Blyth DG, De Sylva DP. Mother's milk turns toxic following fish feast (letter). JAMA 1990; 264:2074.

\section{Revista Biomédica}

